\title{
On some weak separation axioms
}

\section{SABIR HUSSAIN}

\section{ABSTRACT.}

We introduce and explore weak separation axioms namely $\gamma$-semi- $T_{i}$ ( for $\left.i=0,1,2\right)$ spaces. We also define and discuss $\gamma$-semi- $D_{i}($ for $i=0,1,2)$ spaces and develop the relations between these spaces. Moreover, we initiate the concept of $\gamma$-S-continuous function and discuss the behavior of $\gamma$-semi- $D_{1}$ space under $\gamma$-S-continuous function.

Acknowledgement. The author is thankful to the editor-in-chief, Prof. Vasile Berinde for his remarks towards the improvement of this paper.

\section{REFERENCES}

[1] Ahmad, B. and Hussain, S., Properties of $\gamma$-Operations on Topological Spaces, Aligarh Bull. Math., 22 (2003), No. 1, 45-51

[2] Ahmad, B. and Hussain, S., $\gamma$-Semi-Open Sets in Topological Spaces II, Sotheast Asian Bull. Math., 34 (2010), No. 5, 997-1008

[3] Ahmad, B. and Hussain, S., $\gamma$-Convergence in Topological Spaces, Southeast Asian Bull. Math., 29 (2005), $832-842$

[4] Ahmad, B. and Hussain, S., $\gamma^{*}$-Regular and $\gamma$-Normal Space, Math. Today., 22 (2006), No. 1, 37-44

[5] Ahmad, B. and Hussain, S., On $\gamma$-s-Closed Subspaces, Far East Jr. Math. Sci., 31 (2008), No. 2, 279-291

[6] Ahmad, B., Hussain, S. and Noiri, T., On Some Mappings in Topological Space, Eur. J. Pure Appl. Math., 1 (2008), 22-29

[7] Ahmad, B., Hussain, S. and Noiri, T., $\gamma$-Semi-Open Sets in Topological Spaces, Asian Eur. J. Math., 3 (2010), No. 3, 427-433 DOI: $10.1142 /$ S1793557110000337

[8] Ahmad, B., Hussain, S. and Noiri, T., On $\gamma$-Semi Continuous Functions, Punjab University Jr. Math., 42 (2010), 57-65

[9] Hussain, S., On Some Properties of $\gamma$-semi- $R_{0}$ Spaces, , Creat. Math. Inform., 23 (2014), No. 2, 203-207

[10] Hussain, S. and Ahmad, B., Applications of Minimal $\gamma$-Semi-Open Sets, , Fasciculli Mathematici, 46 (2011), 65-76

[11] Hussain, S. and Ahmad, B., On $\gamma$-s-Closed Spaces, Sci. Magna Jr., 3 (2007), No. 4, 89-93

[12] Hussain, S. and Ahmad, B., On $\gamma$-s-Regular Spaces and Almost $\gamma$-s-Continuous Functions, Lobackevskii. J. Math., 30 (2009), No. 4, 263-268. DOI: $10.1134 / 51995080209040039$

[13] Hussain, S. and Alghamdi, M. A., Some Properties of Weak form of $\gamma$-Semi-Open Sets, Tamkang Jr. Math., 42 (2010), 57-65 DOI:10.5556/j.tkjm.43.2012.329-338

[14] Kasahara, S., Operation-Compact Spaces, Math. Japon., 24 (1979), 97-105

[15] Levine, N., Semi-open sets and semi continuity in topological spaces, Amer. Math. Monthly, 70 (1963), 36-41

[16] Levine, N., Generalized closed sets in topological spaces, Rend. Ser. Math. Palerno., (2) 19 (1970), 89-96

[17] Ogata, H., Operations on Topological Spaces and Associated Topology, Math. Japon., 36 (1991), No. 1, 175-184

[18] Umehara, J., Maki, H. and Noir, T., Bi operations on topological spaces and some separation axioms, Mem Fac. Sci. Kochi University Ser. A. Math., 13 (1992), 45-59

[19] Umehara, J., Maki, H. and Noir, T., A certain bi operations on topological spaces, Mem Fac. Sci. Kochi University Ser. A. Math., 15 (1994), $41-49$

DePartMent OF MATHEMATics

COLLEGE OF SCIENCE

QASSIM UNIVERSITY

P. O. BOX 6644, BURAYDAH 51452, SAUdi ARABIA

E-mail address: sabiriub@yahoo.com; sh.hussain@qu.edu.sa 